\title{
MEDIUM RESOLUTION STELLAR SPECTRA IN THE TWO-MICRON REGION
}

\author{
A. R. HYLAND \\ Mount Stromlo and Siding Spring Observatory, Australian National University, Australia
}

\begin{abstract}
The role of medium resolution spectroscopy in the two micron region is discussed. Examples of the spectral features amenable to observation are shown. These include the vibration rotation bands of the important molecules ${ }^{12} \mathrm{C}^{16} \mathrm{O},{ }^{13} \mathrm{C}^{16} \mathrm{O}, \mathrm{H}_{2} \mathrm{O}$ and $\mathrm{CN}$, as well as the $\mathrm{B} \gamma$ line of hydrogen. Band strength-colour and -luminosity relations for the $\mathrm{CO}$ and $\mathrm{H}_{2} \mathrm{O}$ bands have been derived from observations of approximately 100 stars of spectral types later than G5. The interpretation of these and the outstanding problems (such as the colour dependence of the $\mathrm{CO}$ band strengths in carbon stars) are discussed.

Examples of the use of two-micron spectroscopy in the spectral classification of infrared sources, and the infrared components of symbiotic stars, etc., are shown. The extension of this kind of observation to the nuclei of galaxies is briefly discussed in relation to stellar population studies.
\end{abstract}

\section{Introduction}

Recent developments in high resolution Fourier transform spectroscopy in the infrared have led to exciting advances in the observation and interpretation of molecular absorption features in the atmospheres of late type stars. The high resolution obtainable is ideal for the investigation of stellar atomic and isotopic abundances (particularly for the important light elements $\mathrm{C}, \mathrm{N}$ and $\mathrm{O}$ ) and for detailing the physical processes operative within the photospheric layers.

In the present circumstances the role of medium resolution infrared spectroscopy (where we define medium resolution to cover the range 6-24 $\mathrm{cm}^{-1}$ ), has been changed from its former use as a tool in the pioneering investigations of stellar molecular band structure in the 1-4 $\mu$ region (Kuiper 1962; Boyce and Sinton, 1964, 1965; Sinton, 1966; McCammon et al., 1967). In my view, it should be used to provide quantitative information on a relatively small number of important observable quantities (such as the strengths of the $\mathrm{CO}$ and $\mathrm{H}_{2} \mathrm{O}$ bands) for a statistically significant number of stars, and further to extend the use of infrared spectroscopy to the faintest available limits. Such a role parallels that of low resolution spectroscopy at optical and near infrared wavelengths and is typified by the near infrared results presented by Wing (1974).

Discussions of medium resolution infrared spectra in the literature have generally been qualitative in nature, aimed at the identification of molecular bands and the approximate temperature and luminosity dependence of these features, e.g. McCammon et al. (1967); Johnson and Méndez (1970). It is to be expected that future observations will concentrate on the quantitative measurement of the broad observable features and thus be amenable to explicit theoretical interpretations.

In this paper we shall discuss the astrophysical implications of available medium resolution spectra in the limited wavelength range 1.9-2.5 $\mu$. Most of the discussion 
will be based on the extensive observations of Frogel and Hyland in that range (see Frogel, 1971; Frogel and Hyland, 1972; Hyland et al., 1972), made with various telescopes of the Hale Observatories. The particular wavelength range was chosen because of its proximity to both the peak sensitivity of $\mathrm{PbS}$ detectors and the maximum photospheric emission in late type stars, and because it contains absorption bands of the important molecular species $\mathrm{CO}, \mathrm{H}_{2} \mathrm{O}$ and $\mathrm{CN}$. The two major areas covered by this discussion will be:

(a) the observed quantitative empirical relationships between the colour and molecular band strengths for a variety of late type stars and the problems which arise in the interpretation of these relationships, including the determination of the ${ }^{12} \mathrm{C} /{ }^{13} \mathrm{C}$ isotopic abundance ratios from th $\mathrm{CO}$ bands; and

(b) the spectral classification of infrared sources, including the identification of the infrared components of symbiotic stars, spectroscopic binaries and novae.

\section{Observational Techniques}

While observations at medium resolution in the two micron region have been made with both Fourier transform techniques (e.g. Johnson and Méndez, 1970) and conventional grating spectroscopy, we shall be for the main part discussing observations made in the years 1968-1970 using the latter technique by J. Frogel and myself. The system comprised the 0.5-m Ebert-Fastie spectrometer described by McCammon et al. (1967) attached to the 60,100 and 200-in. telescopes of the Hale Observatories. It was a dual beam system with star-sky chop and a monitor channel; the required wavelength range was covered by continuous rotation of the grating, and the output signal was digitized and recorded once a second. Using an entrance aperture of 4-mm provided an essentially slitless mode, while the exit aperture was set at either $0.5 \mathrm{or} 1.0-\mathrm{mm}$ to provide resolutions of $32.5 \AA$ and $65 \AA$ respectively $\left(\sim 6-12 \mathrm{~cm}^{-1}\right)$. The scan rate was adjusted so that the required resolution was achieved.

All spectrophotometric studies, but most especially those in the infrared meet two important calibration problems: (a) the correction of the spectra for terrestrial absorption features $\left(\mathrm{CO}_{2}, \mathrm{H}_{2} \mathrm{O}\right.$ and $\mathrm{CH}_{4}$ are the major contributors in two micron region); and (b) the calibration of the observed fluxes onto an absolute scale so that both the level and shape of the continuum will be correct.

By making the basic assumption that the spectrum of $\alpha$ Lyr is correctly represented by the most recent model atmosphere calculations, (Schild et al., 1971), and that apart from $\mathbf{B} \gamma$ no line absorption features are strong enough to affect the spectrum at the resolutions in question, both (a) and (b) could be achieved. This however necessitated the additional constraint that $\alpha$ Lyr and the program star be observed at the same air mass. In practice, because of the highly saturated nature of many of the terrestrial bands, this constraint could be relaxed, to a limited degree and also, other early type stars such as $\alpha \mathrm{CMa}$ and $\alpha \mathrm{CMi}$ were set up to be secondary standards. Although this method differs from the more rigorous approach of Johnson et al. (1968) (who used lunar spectra from ground based and air-borne observations to define the terrestrial 
absorption), the results have been found to be very similar. Examination of published spectra show that only in few cases is the terrestrial absorption poorly removed.

\section{Features Observable at Medium Resolution}

A detailed discussion of molecular and atomic features observed in the infrared spectra of stars has been given by Spinrad and Wing (1969) and will not be repeated here. We shall concentrate instead on those features which are observable and quantitatively measurable in the two micron region. This implies that a feature must necessarily have an equivalent width $\geqslant 6 \AA$ for it to be measurable with reasonable precision (say $10 \%$ ). The exact value necessarily depends on the shape of the feature, i.e. whether it is a molecular band, a single atomic line or blend of atomic lines. There are a few features which fulfill these requirements, and these will be considered here.

\subsection{MOLECULAR BANDS}

\subsubsection{Carbon Monoxide}

The first overtone $(\Delta v=2)$ vibration-rotation band sequence of ${ }^{12} \mathrm{C}^{16} \mathrm{O}$ has six vibrational band heads between 2.29 and $2.45 \mu$. These are visible in all giant stars with spectral types later than G5, and have been seen by a large number of observers at both low and medium resolution, see e.g. Boyce and Sinton $(1964,1965)$; Mertz (1965); Moroz (1966); McCammon et al. (1967); Johnson et al. (1968). Typical spectra of $M$ stars showing the sequence are given in Figure 1, where five band heads are easily visible. The bands are clearly amenable to precise measurement as long as the continuum shape can be reasonably defined.

In addition the isotopic molecule ${ }^{13} \mathrm{C}^{16} \mathrm{O}$ has visible band heads in most giant stars later than $\mathrm{K0}$, the first three of these are indicated in Figure 1 and are easily seen in the spectrum of $\mu \mathrm{Gem}$.

The importance of $\mathrm{CO}$ hinges on the fact that it is essentially totally associated in all cool stars and thus in principle the band strength provides a hold on the atomic abundance of the less abundant species. It is therefore an important link in the determination of $\mathrm{C}, \mathrm{N}, \mathrm{O}$ abundances in late type stars. The presence of the bands of ${ }^{13} \mathrm{C}^{16} \mathrm{O}$ further provides an opportunity to derive the ${ }^{12} \mathrm{C} /{ }^{13} \mathrm{C}$ abundance ratio for comparison with theoretical predictions.

\subsubsection{Water}

The absorption bands of water vapour $\left(\mathrm{H}_{2} \mathrm{O}\right)$ which have their peak absorption near 1.9 and $2.7 \mu$ as in the terrestrial atmosphere, also occur in very cool stars. At stellar temperatures however, the bands are broadened by the increased strengths of lines from excited levels and extend well into the two micron window. The spectrum of U Ori (M8e) shown in Figure 1 provides a striking example of $\mathrm{H}_{2} \mathrm{O}$ absorption between 2.0 and $2.2 \mu$. The same authors who observed $\mathrm{CO}$ have reported observations of the water vapour (steam) absorption bands in M type Mira variables, although 




Fig. 1. Infrared spectra of $\mu$ Gem (M3 III) and the long period variable star U Ori (M8e) between 1.95 and $2.45 \mu$ are shown. The resolution is $32.5 \AA$ and the ordinate is the log of the flux per unit wavelength interval plus a constant. The prominent band heads of ${ }^{12} \mathrm{C}^{16} \mathrm{O}$ and ${ }^{13} \mathrm{C}^{16} \mathrm{O}$ are identified.

Note the deep absorption of $\mathrm{H}_{2} \mathrm{O}$ in $\mathrm{U}$ Ori at wavelengths shorter than $2.2 \mu$.

no quantitative estimates were made before the work of Frogel (1970). It is obvious from Figure 1 that at medium resolution the $1.9 \mu$ band appears as a source of continuous opacity. While measurement of total absorption can be made from the available spectra, a water vapour index (WV) as defined by Frogel (1971) (See Section 4) appears to be a good measure of the strength of the $1.9 \mu$ band, which may be compared with model atmosphere predictions (Auman, 1969).

\subsubsection{Cyanogen}

The extensive red system of cyanogen $(\mathrm{CN})$ has several band heads in the two micron region. The $R_{21}, R_{22}, R_{11}$ and $Q_{11}$ band heads of the $(2,4)$ and $(3,5)(\Delta v=-2)$ systems were first positively identified in the spectra of carbon stars by Thompson and Schnopper (1970). The identification of the corresponding $(1,3)$ band heads of same system in carbon stars was made by Frogel and Hyland (1972). At medium resolution these $\mathrm{CN}$ bands are only visible in the spectra of carbon stars. Because of the large number of individual lines over a wide wavelength range the $\mathrm{CN}$ absorption behaves like a continuous opacity in a manner similar to $\mathrm{H}_{2} \mathrm{O}$. Considerable interest has recently been shown in the prediction of CN band strengths (Johnson et al., 1972; 
Alexander and Johnson, 1972) and the incorporation of $\mathrm{CN}$ opacities into realistic stellar atmosphere models. Thus although it is quantitatively more difficult to measure $\mathrm{CN}$ band strengths, the likely availability of synthetic spectra and the importance of $\mathrm{CN}$ in the atmospheres of carbon stars underline the necessity to consider $\mathrm{CN}$ seriously.

In Figure 2 the $\mathrm{CN}$ band heads are easily identified in the carbon star, U Hya $(\mathrm{C} 7,3)$ but are much less prominent in the long period variable carbon star $\mathrm{V} \mathrm{CrB}$ $(\mathrm{C} 6,3)$. Very weak $\mathrm{CN}$ absorption is probably present in the S star AA Cyg, which has, on the other hand, among the strongest $\mathrm{CO}$ bands measured.

\subsubsection{Molecular Features not Seen}

Among other molecules, high excitation vibration-rotation bands of $\mathrm{TiO}, \mathrm{SiO}$ are predicted to lie in the two micron region, but have not definitely been identified at the resolutions in question. Similar comments can be made regarding $\mathrm{HCN}$ and $\mathrm{C}_{2} \mathrm{H}_{2}$ whose predicted band heads are shown in Figure 2. Finally the predicted quadrupole

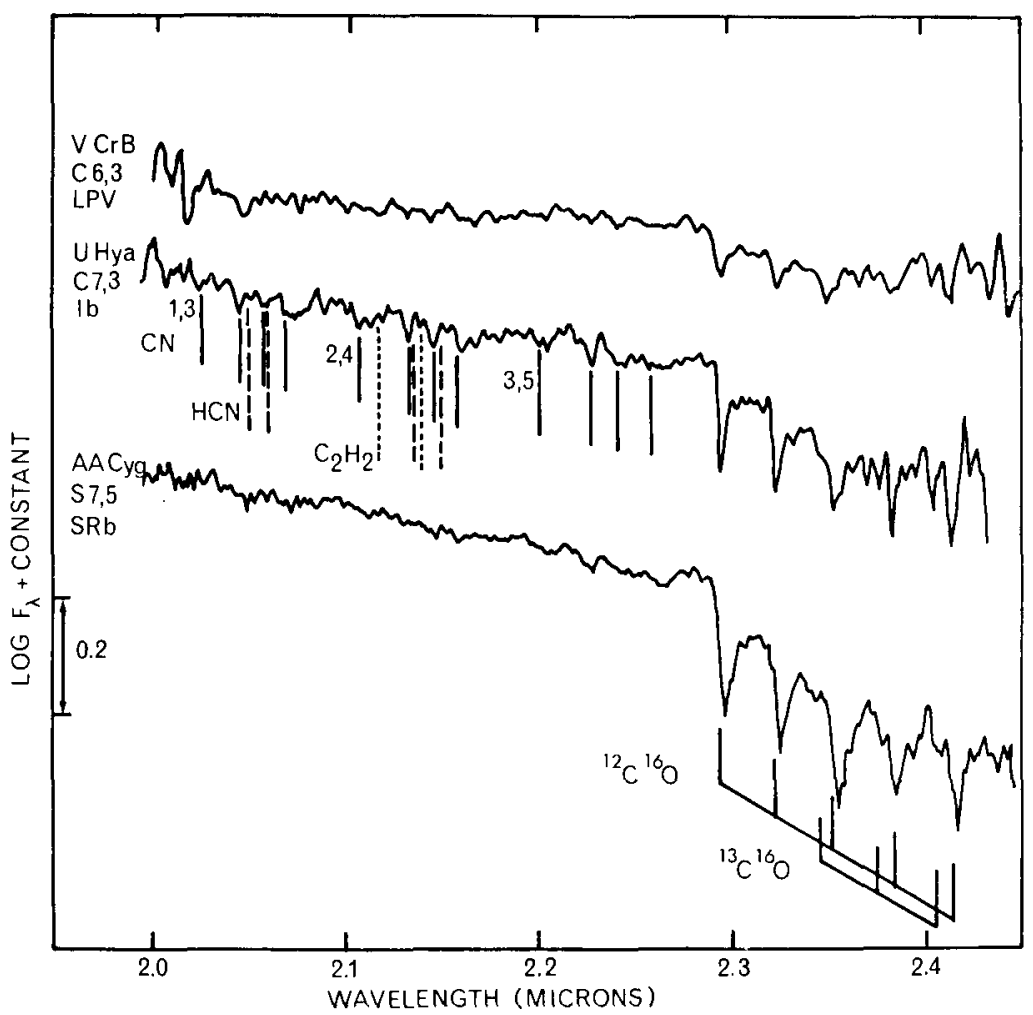

Fig. 2. Infrared spectra of the long period variable carbon star, $\mathrm{V} \mathrm{CrB}$, the irregular variable carbon star $\mathrm{U}$ Hya, and the S star AA Cyg are shown. In addition to the band heads of ${ }^{12} \mathrm{C}^{16} \mathrm{O}$ and ${ }^{13} \mathrm{C}^{16} \mathrm{O}$, the positions of the identified band heads of the red system of $\mathrm{CN}$ are shown, as are the predicted positions for bands of $\mathrm{HCN}$ and $\mathrm{C}_{2} \mathrm{H}_{2}$. The $\mathrm{CO}$ bands in the $\mathrm{S}$ star are among the strongest yet observed. Note the difference in the strengths of the $\mathrm{CO}$ bands in the two carbon stars. 
line of $\mathrm{H}_{2}$ (Spinrad and Wing, 1969) has been searched for unsuccessfully both at medium and high resolution.

\subsection{ATOMiC FeAtures}

In the remainder of this paper we shall be considering primarily measurements of the molecular species described above, largely because the majority of work has been done in that area. There are, however, several important atomic features for which some data is available, and which should be included in this discussion.

\subsubsection{Hydrogen}

The only measurable feature due to atomic hydrogen in the $2.0-2.5 \mu$ region is the Brackett $\gamma(\mathrm{B} 7)$ transition at $2.1655 \mu$. It is the major atomic feature in the region and a number of measurements have been reported in the literature. The strength of the line in absorption is such that it is measurable in stars with spectral types ranging from $\mathbf{B}$ to $\mathrm{K}$, and its temperature dependence makes it a useful spectral type indicator for the earlier spectral classes.

The B7 line has also been observed in emission in the spectra of diffuse H II regions and planetary nebulae (Hilgeman, 1970), $\eta$ Car (Neugebauer and Westphal, 1968) and in P Cyg and novae (Hyland, 1973). Knowledge of the emission strength of B7 is particularly helpful in deducing reddening estimates for these sources.

\subsubsection{Helium}

There is little available information on the occurrence of helium lines in the $2-2.5 \mu$ region of the spectrum. No measurements of absorption lines have been reported. However, the $1 s 2 s-1 s 2 p$ transition of neutral helium at $2.05813 \mu$ has been observed in emission in diffuse nebulae and planetary nebulae (Hilgeman, 1970) and is also present in the spectra of P Cyg and Nova Aql 1970 (Hyland, 1973). This line is also useful for reddening estimates and for the determination of $\mathrm{He} / \mathrm{H}$ abundance ratios in these stars. It appears that there is considerable scope for wider investigations of these lines.

\subsubsection{Other Atomic Lines}

An investigation of the features observed in the spectra of $\mathrm{K}$ and $\mathrm{M}$ giants reveals several which may be identified with blends of closely spaced atomic lines of Si I, Mg I and Ti I (Frogel, 1971), given by Spinrad and Wing (1969). These show a systematic trend with spectral type, becoming stronger with increasing lateness of spectral type. The strengths are however all $\leqslant 3 \AA$ and hence do not lend themselves to precise measurement such as we are discussing here.

\section{Empirical Band Strength - Colour Relationships}

In this section we shall discuss the measured $\mathrm{CO}$ and $\mathrm{H}_{2} \mathrm{O}$ band strengths from observations of approximately 100 late type stars covering the spectral types G5-M8 and 
including the long period variable $\mathrm{M}$ stars, $\mathrm{S}$ stars and carbon stars. The large number of observations provides the opportunity for displaying the overall relations rather than the spectra of individual stars as is necessary for high resolution observations. The band strength of $\mathrm{CO}, \mathrm{W}(\mathrm{CO})$, is defined to be the equivalent width of the $\mathrm{CO}$ absorption between 2.29 and $2.39 \mu$, i.e. between the $(2,0)$ and $(5,3)$ band heads of ${ }^{12} \mathrm{C}^{16} \mathrm{O}$. As can be seen in Figure 1 this is contaminated by the $(2,0)$ band of ${ }^{13} \mathrm{C}^{16} \mathrm{O}$, although the effect is slight.

The strength of the $1.9 \mu$ water vapour band is estimated by the use of the index WV, which is defined to be (after Frogel, 1971)

$$
\log \frac{F_{\text {cont }}(\lambda=2.10)}{F_{\lambda}(\lambda=2.10)}
$$

which differs slightly from the definition by Frogel (1970). $F_{\text {cont }}(\lambda=2.10)$ is the predicted continuum flux at $2.10 \mu$ obtained from extrapolation of the continuum in the manner shown in Figure 1.

\subsection{Carbon monoxide in G5-M8 Giants and Supergiants}

It is clear from the results of two micron spectroscopy over the last few years that the $\mathrm{CO}$ band strengths increase with increasing lateness of spectral type (e.g. Johnson and Méndez, 1970). There are several relevant questions which may be asked: (1) Are the $\mathrm{CO}$ band strengths sufficiently sensitive to be used for temperature determination, and how can luminosity effects be taken into account? (2) Is there evidence for abundance variations in the observed $\mathrm{CO}$ band strengths? This is of particular interest for the metal poor $\mathrm{K}$ giants and for the super metal rich stars, for abundance determinations may help define the relationship between the abundances of the light elements, $\mathrm{C}, \mathrm{N}$ and $\mathrm{O}$, and the heavy metals. (3) Can the observed strengths of the $\mathrm{CO}$ bands in the various species of late type star be explained theoretically? (4) What ${ }^{12} \mathrm{C} /{ }^{13} \mathrm{C}$ abundance ratios are derived from the ${ }^{12} \mathrm{C}^{16} \mathrm{O}$ and ${ }^{13} \mathrm{C}^{16} \mathrm{O}$ band strengths, and how certain are these.

In the following paragraphs and in Sections 4.2 and 4.3, we will consider how the observations bear on these questions.

In Figure 3 the $W(\mathrm{CO})$ strengths of G5-M8 giants are plotted as a function of $(J-L)$. The $(J-L)$ colour is known to be a good temperature indicator for cool stars when the interstellar reddening is small, as is the case for the bright stars in this discussion. The tight relationship of $W(\mathrm{CO})$ and $(J-L)$ may thus be interpreted in terms of the temperature dependence of $W(\mathrm{CO})$. The line in Figure 3 is a least square straight line fit to the data. Since $\mathrm{CO}$ is fully associated for all temperatures lower than $\sim 4000 \mathrm{~K}$, the increase in strength with decreasing temperature is not due to the increasing number density of $\mathrm{CO}$ molecules but rather to the decrease in the number density of $\mathrm{H}^{-}$which provides the continuous opacity. Even so it has been necessary to invoke high microturbulent velocities $\left(\sim 10 \mathrm{~km} \mathrm{~s}^{-1}\right)$ to obtain theoretical fits to the $\mathrm{CO}$ band strengths. These velocities are considerably higher than those obtained by studies 


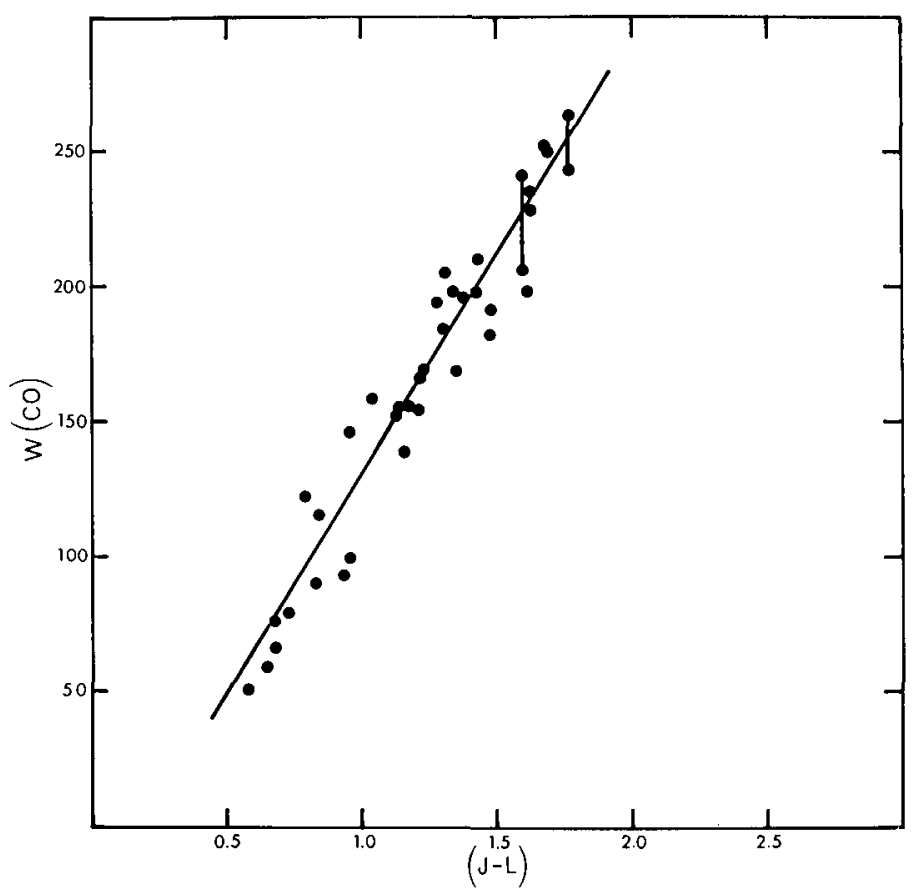

Fig. 3. The observed relation between $W(\mathrm{CO})$, as defined in the text, and the $(J-L)$ colour, for G5-M8 giants. The continuous line is the least square best fit straight line to the observations. The lines joining the dots refer to different spectroscopic observations of the same stars.

of optical spectra, and the relationship of the turbulence parameters in the optical and infrared are worthy of investigation at high resolution.

The equivalent observations for supergiant stars are shown in Figure 4, where the line is that derived for the giant stars. It is clear that at a given colour (i.e. temperature) the supergiants have stronger $\mathrm{CO}$ bands than the giants. The exact relationship is unfortunately clouded by the presence of strong interstellar and circumstellar absorption, for several of the supergiants. It appears that there is no easy two dimensional (luminosity, temperature) classification possible unless the colour is also known. The continuum slope obtained directly from the spectra can be used in place of $(J-L)$, and partly obviates the necessity for broad band measurements. Unfortunately the precision with which the slope can be measured is much lower than that obtainable with broad band measurements.

Qualitative inspection of giant and supergiant spectra reveals a fundamental difference in the shape of the $\mathrm{CO}$ absorption bands, however, no quantitative description of this difference has been proposed. Inspection of the $\mathrm{CO}$ bands in $\mu \mathrm{Gem}$ (Figure 1), IRC +20439 (Figure 8) which are both M giants, and in the S star AA Cyg (Figure 2) (whose spectrum is identical to $\mathrm{M}$ supergiants) will illustrate the above point.

One further remark should be made about Figure 3. The spread in the observations at a given colour is larger in the early $\mathrm{K}$ stars $(J-L \sim 1.0)$ than would be expected from 


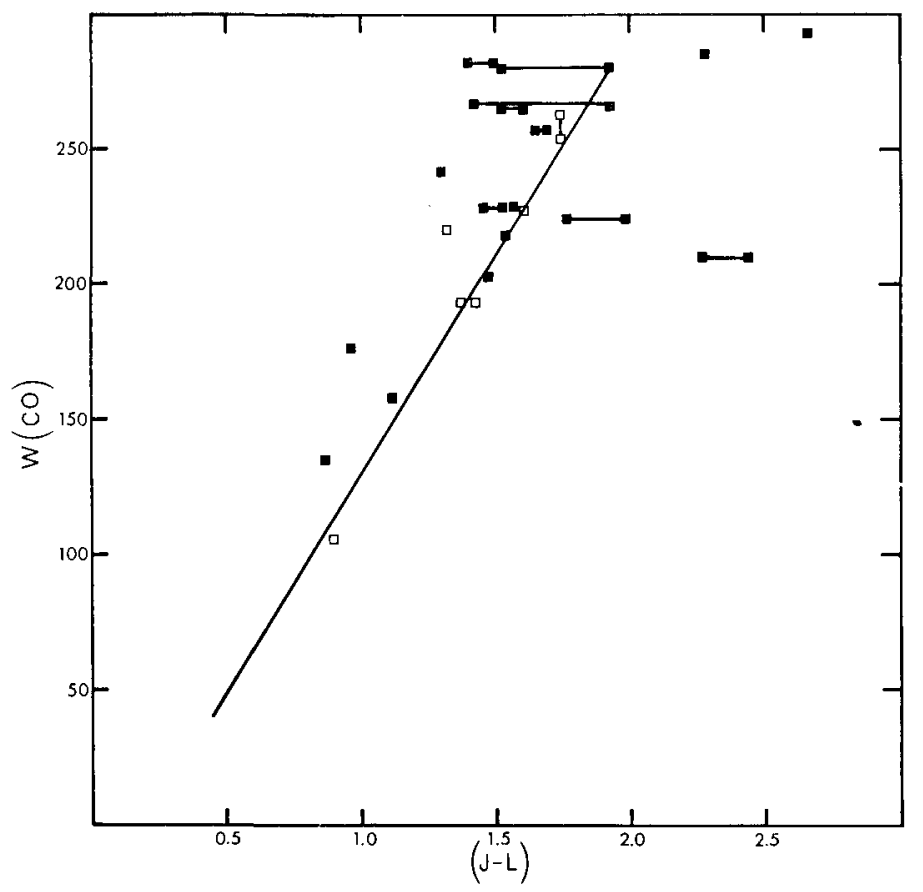

Fig. 4. The same as for Figure 3 for supergiant stars. The open squares are class II stars, while the filled squares refer to luminosity classes Ib, Iab and Ia. The straight line is the mean giant relation as shown in Figure 3. The straight lines joining the filled squares refer to independent measurements of the stellar colours.

the observational errors $( \pm 15 \AA)$. It is probable that these differences are due to real differences in the abundances in these stars. This is just that region of temperature in which the Cannon (1970) clump stars are found, and where spectral peculiarities such as strong $\mathrm{CN}$ become apparent. The correlation of $\mathrm{CO}$ band strength (which depends mainly on the abundance of carbon in these stars) with parameters such as $\mathrm{CN}$ or metal line strength may provide important clues as to the evolutionary status of early $\mathrm{K}$ giant stars.

\subsection{Carbon monoxide band Strengths in long period variable M stars, S stars AND CARBON STARS}

In Figure 5 the observed $W(\mathrm{CO}),(J-L)$ relationships for all common species of late type giant star are presented. Omitted from this figure are the supergiants and the dwarf stars to minimise confusion. We note the following points:

(1) The long period variable $\mathrm{M}$ stars exhibit large cyclical variations in $W(\mathrm{CO})$. The arrow indicates a typical excursion of the band strength. At maximum $W(\mathrm{CO})$ the long period variable $M$ stars an extension of the normal giant line to larger $W(\mathrm{CO})$ and redder $(J-L)$.

(2) As a group the $\mathrm{S}$ stars have the strongest $\mathrm{CO}$ bands measured, being typical of, 
and stronger than those of the $\mathrm{M}$ supergiants. This effect is probably due in part to the decrease in continuous opacity through the lack of $\mathrm{H}_{2} \mathrm{O}$ absorption (see Section 4.4). Whether the $\mathrm{S}$ stars as a group have gas pressures more similar to supergiants than giants is not clearly established.

(3) Values of $W(\mathrm{CO})$ in the semi-regular and irregular carbon stars show a definite decrease with increasing $(J-L)$ colour (Frogel and Hyland, 1972), from values typical of late $\mathrm{M}$ stars at $(J-L) \sim 2.0$ to values typical of $\mathrm{K}$ stars at $(J-L) \sim 2.6$. The long period variable carbon starts cover an even larger range of $W(\mathrm{CO})$ values as illustrated in Figure 5, but are all much redder than the irregular and semi-regular carbon stars.

Of the above three remarks, the carbon star sequence is probably the most interesting, and the least understood. We shall therefore discuss in further detail the problems raised by the carbon star observations.

Several interesting correlations have been established by Frogel and Hyland (1972), the three most important being:

(1) the $\mathrm{CO}$ strength decreases with increasing colour, both in $(J-L)$ and the (3.5-8.4 $\mu$ ) colour;

(2) the $\mathrm{CO}$ strength decreases with increasing carbon parameter;



Fig. 5. The observed $W(\mathrm{CO})-(J-L)$ relation for various classes of cool stars including the normal giants, S stars, the long period variable (LPV) M stars, the irregular and semi-regular carbon stars, and the LPV carbon stars. 
(3) the CO strength increases with decreasing temperature as determined from optical temperature parameters.

The last of these will be dealt with first. The temperature scale of the carbon stars is a rather controversial topic at the present time. From $(R-I)$ colours Eggen (1972) has proposed that all the carbon stars lie in the very restricted temperature range $3300-3500 \mathrm{~K}$. Bessell and Lee (1972) from infrared stars of a similar group conclude that the blackbody continuum temperatures are lower than those of Eggen by $\sim 500 \mathrm{~K}$ and that the spread of temperatures is somewhat larger. Unfortunately the broad band infrared colours (Mendoza and Johnson, 1967; Frogel and Hyland, 1972) only serve to confuse the issue because of the definite effects of circumstellar grain emission in many cases although the colour temperatures agree better with the scanner results. It is clear also that the effect of the atomic abundances on the atmospheric opacities is to alter the colour-temperature relation for the carbon stars. Until a definitive calibration of colour against temperature can be achieved via, for instance, lunar occultation measurements of carbon stars or realistic model atmospheres replace black body colours, this problem will remain one of the major obstacles in the study of carbon stars.

From (1) and (2) it follows that the $(J-L)$ colour also increases with increasing carbon parameter, which is an important clue as to the relevant mechanism involved. Two possibilities may be suggested to explain (1) and (2). Either (a) the abundance of oxygen is decreased as that of carbon is increased, while the major opacity source in the region of the $\mathrm{CO}$ bands is unchanged, or (b) as the carbon abundance is increased, the continuous opacity in the region of the $\mathrm{CO}$ bands increases, so that, although the number density of $\mathrm{CO}$ remains sensibly the same, the equivalent width of the $\mathrm{CO}$ bands decreases. The former suggestion is hard to reconcile with any process of mixing nuclear burning products to the surface, since it requires a decrease in the abundance of oxygen relative to $\mathrm{H}^{-}$(which is assumed in this explanation to be the major opacity source).

The latter suggestion appears to be much more likely, but it requires that $\mathrm{CN}$ be the major opacity source in the vicinity of the CO bands, and that it increases with increasing abundance of carbon. Unfortunately the observations do not provide clear evidence of the predicted increase in the strength of the $\mathrm{CN}$ bands between 2.0 and $2.3 \mu$ with increasing carbon parameter (see Frogel and Hyland, 1972). However, if these bands provide the major continuous opacity in that region also, such an effect would undoubtedly be masked.

The predictions of realistic model atmospheres could solve this problem. For example, if atmospheres with constant oxygen abundance and increased $\mathrm{C} / \mathrm{O}$ have (a) redder broad band colours and (b) weaker apparent $\mathrm{CO}$ strengths, then the above suggestion would be a very plausible explanation. Certainly the explanation of the carbon star $\mathrm{CO}$ band strengths in terms of the absolute abundances of $\mathrm{C}$ and $\mathrm{O}$ poses crucial tests for the nuclear processes (e.g. the triple- $\alpha$ reaction or the CNO bi-cycle) proposed to explain the properties of carbon stars. Although some evidence supported the CNO bi-cycle hypothesis (Thompson et al., 1971), recent more detailed analyses of 
molecular abundances in carbon stars appear to exclude it (Thompson, 1974). Thus the present emphasis lies in the comparison of the results of the triple- $\alpha$ process with the observations.

The position of the long period variable carbon stars relative to the irregular and semi-regular carbon stars in Figure 5 has been interpreted in terms of increased circumstellar dust shell characteristics in the long period variables (Frogel and Hyland, 1972) due to pulsationally coupled mass loss. This proposal remains an attractive and plausible explanation. The reason for carbon grain emission strongly affecting the two micron spectral region, whereas silicate emission does not (see Section 5.1) is that carbon grains can exist to much higher temperatures (i.e. $2000 \mathrm{~K}$ ) than the silicates, and thus emit strongly at wavelengths down to $1 \mu$.

Many problems related to the evolutionary status and physical phenomena associated with carbon stars remain unsolved. We have seen that two micron spectroscopy is a valuable addition to the observational techniques available, which raises special problems of its own. However the recent upsurge of interest in carbon stars, both theoretical and observational, should result in major advances in our understanding of the subject over the next few years.

\subsection{The ISOTOPIC ${ }^{12} \mathrm{C} /{ }^{13} \mathrm{C}$ ABundance Ratio}

The presence of both ${ }^{12} \mathrm{C}^{16} \mathrm{O}$ and ${ }^{13} \mathrm{C}^{16} \mathrm{O}$ band heads in the $2.3-2.5 \mu$ region allows one to obtain the ${ }^{12} \mathrm{C} /{ }^{13} \mathrm{C}$ ratio via analysis of the band strengths. The major problem in the derivation is the unknown degree of saturation of the ${ }^{12} \mathrm{C}^{16} \mathrm{O}$ bands. While the degree of saturation becomes increasingly important at later spectral types, and is thought to be very high in the M supergiants (Thompson et al., 1972), it appears, at least for early $\mathrm{K}$ stars, that it is low enough for realistic abundance ratios to be obtained. The derived values of ${ }^{12} \mathrm{C} /{ }^{13} \mathrm{C}$ for the early K giants (Frogel, 1971) reveal two interesting results. Firstly the majority of early $\mathrm{K}$ giant stars have values of ${ }^{12} \mathrm{C} /{ }^{13} \mathrm{C}$ in the range $5-10$, which is markedly different from the solar and terrestrial value. Secondly, a few stars have values of ${ }^{12} \mathrm{C} /{ }^{13} \mathrm{C}$ much higher than the average. In particular the value for $\beta \mathrm{Gem}$ is $>30$, and for $o^{\prime} \mathrm{CMa}>25$.

We are forced to conclude that real differences in ${ }^{12} \mathrm{C} /{ }^{13} \mathrm{C}$ exist between stars of similar spectral type, and suggest that the exact ratio is critically dependent on the evolutionary history of the individual star. It should be emphasized again, that the early $\mathrm{K}$ giant region is where a large number of spectral peculiarities first become evident. For example the strong CN stars, the weak line stars, the SMR stars and extreme Population II stars all populate the early $\mathrm{K}$ giant region. The causal connection of these peculiarities to the 'clump', asymptotic branch stars has yet to be established. The correlation of the observed ${ }^{12} \mathrm{C} /{ }^{13} \mathrm{C}$ ratio with anyone of these peculiarities would be of considerable interest in this regard.

\subsection{WATER VAPOUR BAND STRENGTHS IN LATE-TYPE STARS}

The importance of water vapour absorption in the $2.0-2.5 \mu$ region in very cool stars can be judged from the spectrum of U Ori shown in Figure 1. A quantitative 




Fig. 6. The empirical colour $-\mathrm{H}_{2} \mathrm{O}$ index $(W V)$ relation for late type stars. The long period variable $M$ stars at minimum $W V$ form an extension of the normal non variable giant relation to redder colours. At maximum $W V$ the long period variable $M$ stars can be clearly distinguished by the strength of their $\mathrm{H}_{2} \mathrm{O}$ absorption.

measure of the strength of the $1.9 \mu$ band (WV) has been defined previously (Section 4). The observed relation of $W V$ to the $(J-L)$ colour for both normal giants and long period variable $M$ stars is shown in Figure 6. This shows that absorption due to the $1.9 \mu$ band of $\mathrm{H}_{2} \mathrm{O}$ becomes important only for stars with $(J-L)>1.5$, i.e. with spectral types later than M6. Thus the presence of $\mathrm{H}_{2} \mathrm{O}$ absorption in the spectrum of a star allows an upper limit to its temperature to be derived.

The position of $M$ supergiants has not been shown in Figure 6, however, with the exception of the late supergiants VY CMa, NML Cyg, no $\mathrm{H}_{2} \mathrm{O}$ absorption is seen, in agreement with molecular abundance calculations.

The $\mathrm{H}_{2} \mathrm{O}$ index $(W V)$ is greatest in the long period variable $M$ stars and may be used to identify such sources. At minimum light, the $\mathrm{H}_{2} \mathrm{O}$ index is larger for all long period variable $\mathrm{M}$ stars observed (Frogel, 1971) than in any of the irregular or non 
variable $\mathrm{M}$ giants. On the other hand, near maximum light (minimum $\mathrm{H}_{2} \mathrm{O}$ strength) the values of $W V$ merely form an extension of the normal giant relationship, albeit to larger values of $(J-L)$. One may define unambiguous spectral types in terms of the $\mathrm{H}_{2} \mathrm{O}$ strength extending as far as M10 in similar manner to the TiO + VO relation of Wing (1967) however, there is no clear correlation of $\mathrm{H}_{2} \mathrm{O}$ band strength with colour temperature. It appears that the band strength-temperature relation may in fact be multivalued. For a full understanding of the cyclical variations of the $\mathrm{H}_{2} \mathrm{O}$ and CO band strengths in long period variable stars, the work of Frogel (1971) needs to be extended considerably.

Almost all of the $\mathrm{S}$ stars, both long period variables and non variables show no evidence for $\mathrm{H}_{2} \mathrm{O}$ absorption, in agreement with the suggestion that $\mathrm{O} / \mathrm{C}=1$ in $\mathrm{S}$ stars. One exception to this rule is $\mathrm{W}$ And, whose $2.0-2.5 \mu$ spectrum is remarkably similar to that of the long period variable $M$ star, $U$ Ori. If $W$ And has true $S$ characteristics, in relation to the strength of its $\mathrm{ZrO}$ bands, it would appear that most $\mathrm{S}$ stars have been subject to two different processes, one which affects only the $\mathrm{O} / \mathrm{C}$ ratio and the other which determines the abundance of the $s$-process elements. Furthermore, the existence of W And can be regarded as evidence that the $s$ process alone has occurred. The possibility which this raises, that the abundance peculiarities in S stars are produced sequentially in two distinct phases, is worthy of full scale investigation.

\section{Further Uses of Two-Micron Spectroscopy}

In the previous sections we have discussed the observations and interpretation of the major molecular features observed between $1.9 \mu$ and $2.5 \mu$ in a variety of late type stars. By utilisation of the above results, however, two micron spectroscopy can make a valuable contribution to a number of other areas of investigation.

\subsection{SPECTRAL CLASSIFICATION OF INFRARED OBJECTS}

Since the advent of the Two Micron Sky Survey (Neugebauer and Leighton, 1969) it has become clear that there are a large number of objects which radiate strongly in the infrared but which are either very faint or totally obscured visually. In the circumstances medium resolution infrared spectroscopy provides the means whereby the nature of the source can often be precisely ascertained. From the empirical band strength relationships discussed above it is possible to derive the abundance, temperature and luminosity characteristics of the source from the spectra alone. This method has proved extremely successful in the case of the infrared source NML Cyg (Johnson, 1968) and in the classification of the visually faint OH/IR stars (Hyland et al., 1969, 1972). From the spectra NML Cyg was classified as an M supergiant, and the $\mathrm{OH} / \mathrm{IR}$ sources as either $\mathrm{M}$ supergiants or long period variable $\mathrm{M}$ stars. Effective temperatures of these sources were determined by comparison with the temperatures of unreddened sources with similar two micron spectra. The validity of this procedure is dependent on the amount of distortion of the molecular $\mathrm{CO}$ and $\mathrm{H}_{2} \mathrm{O}$ features by circumstellar dust emission. It has been shown that such an effect is only marginal 


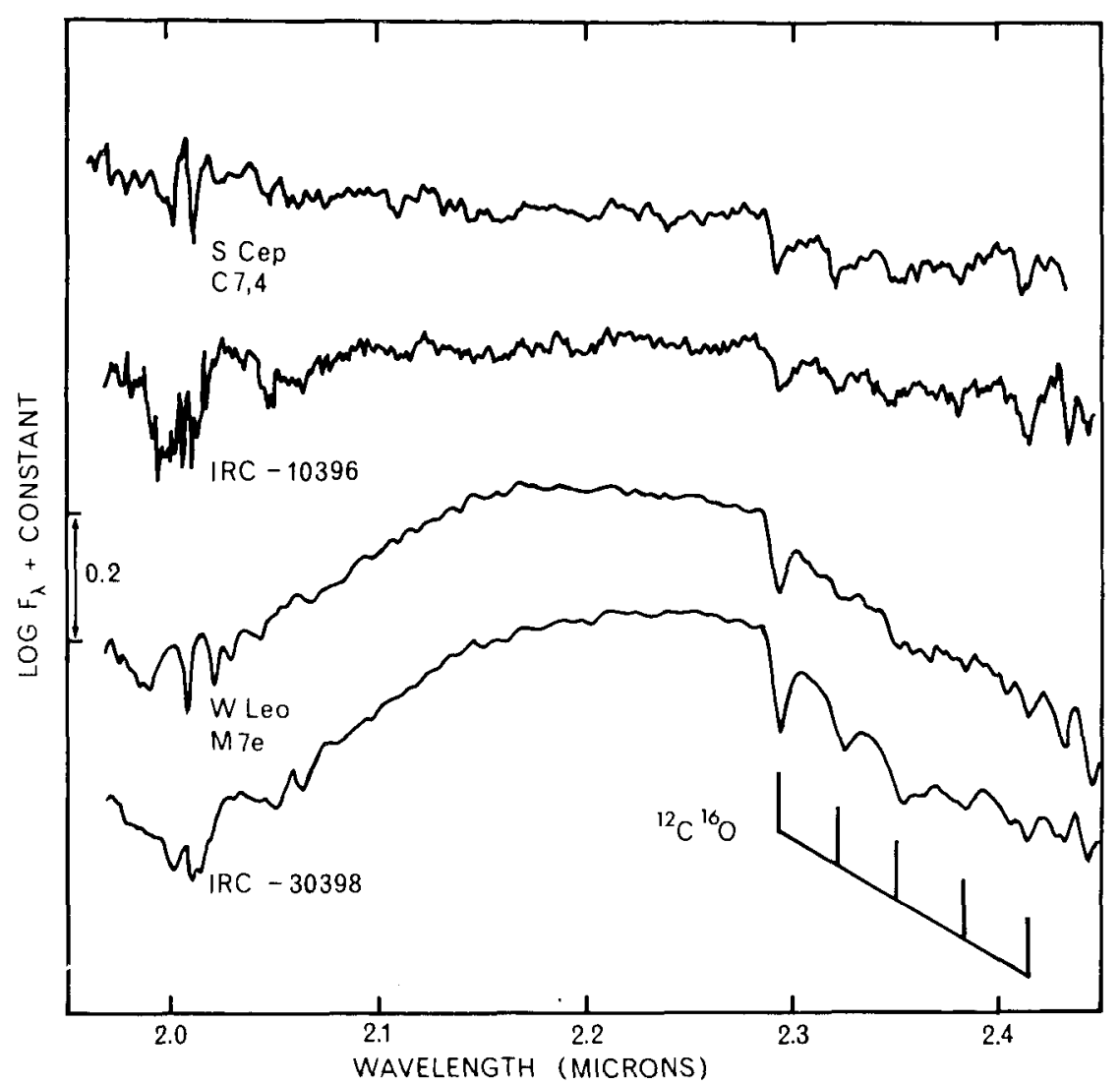

Fig. 7. Two micron spectra of the two infrared sources IRC -10396 and IRC -30398 are compared with spectra of the well known carbon star S Cep $(C 7,4)$ and long period variable $M$ star W Leo $(\mathrm{M} 7 \mathrm{e})$ respectively. Note the remarkable similarity of the spectra of the infrared sources to those of the comparison stars.

even in the case of an optically thick shell such as surrounds VY CMa (Hyland and Gingold, 1972) and hence that in the case of $M$ stars the procedure is both valid and valuable. On the other hand it has been suggested as in 4.2 (Frogel and Hyland, 1972) that the weakening of the $\mathrm{CO}$ absorption in the reddest carbon stars is due to circumstellar emission from carbon grains which may exist at higher temperatures than the silicate like particles found in the shells surrounding $\mathrm{M}$ stars. Indeed the $\mathrm{CO}$ bands in IRC +10216 , the most extreme carbon star known, are almost totally obliterated and the spectrum smooth and featureless (Becklin et al., 1969).

As examples of spectral classification of infrared objects using two micron spectroscopy, the spectra of an oxygen rich $M$ type long period variable W Leo (M7e), and the well known long period variable carbon star S Cep $(C 7,4)$, are compared in Figure 7 with the observed spectra of two infrared objects IRC $-30389(\mathrm{OH} / \mathrm{IR})$, and IRC -10396 respectively. The strength of the $\mathrm{CO}$ and $\mathrm{H}_{2} \mathrm{O}$ bands in W Leo and IRC -30398 , and the strength of the CO and CN bands in S Cep and IRC -10396 
are remarkably similar and demonstrate how well such spectra can be matched with those of 'standard spectroscopic sources'. The only noticeable difference in each case lies in the slightly different continuum slope, which presumably results from the selective nature of circumstellar absorption and emission processes.

\subsection{IDENTIFICATION OF THE INFRARED COMPONENTS OF SYMBIOTIC STARS, NOVAE, Be} STARS, ETC.

There are several categories of stars which appear to consist of a hot and a cool component in close proximity. The problem in these cases is to identify the nature of the cool component, which dominates the infrared spectrum. In some cases clues as to the nature of the cool component are obtained from spectra in the red, where molecular band structure of TiO may be seen. The optical spectrum is of course dominated by the emission of the hot component.

Symbiotic stars are prototypes of this type of object, and in several cases the optical spectra have been sufficient for cool companions of spectral type $\sim \mathrm{M} 2$ to be identified. There are however a number of cases for which the presence of a cool companion has been suspected but whose nature has not been established. It is in these instances that infrared spectra in the two micron region are particularly valuable, and allow for an unambiguous classification of the infrared continuum. This type of observation is also applicable to the infrared excesses observed in novae, P Cyg stars, Be stars and the supergiant $F$ and $G$ stars. We shall discuss here only the observations of four sources as examples of what is seen. The observed 2.0-2.5 $\mu$ spectra are shown in Figure 8.

\subsubsection{The Composite Star IRC +20439}

This source has been identified in the Two Micron Sky Survey with the eighth magnitude A2 star DM $+22^{\circ} 3840$. Close investigation has shown that the two sources are one and the same and spectra in the red (5500-7000 $\AA$ ) confirm the presence of a late type companion whose spectral type is not unambiguously determined. The spectrum of IRC +20439 (in Figure 8 ) with $W(\mathrm{CO}) \sim 250 \AA$ allows us to identify the infrared component as an M7 giant with a temperature close to $2600 \mathrm{~K}$. A report on similar observations of the infrared spectra of symbiotic stars is in preparation. Of particular interest is the unambiguous identification of the infrared component of XX Oph as a late $\mathrm{M}$ star.

\subsubsection{Z CMa}

This is an interesting early type star associated with nebulosity and apparently embedded in dust. It has been studied at infrared wavelengths out to $20 \mu$ (Gillett and Stein, 1971) and discussed by Neugebauer et al. (1971). Without infrared spectra, the possibility that it is a composite star with a late $\mathrm{M}$ component could not be discounted. The two micron spectrum shown in Figure 8 (Racine et al., 1971) shows that there is no evidence for the presence of a late type companion, but that the emission appears to be that of a smooth dust component. The peak in the continuous energy 


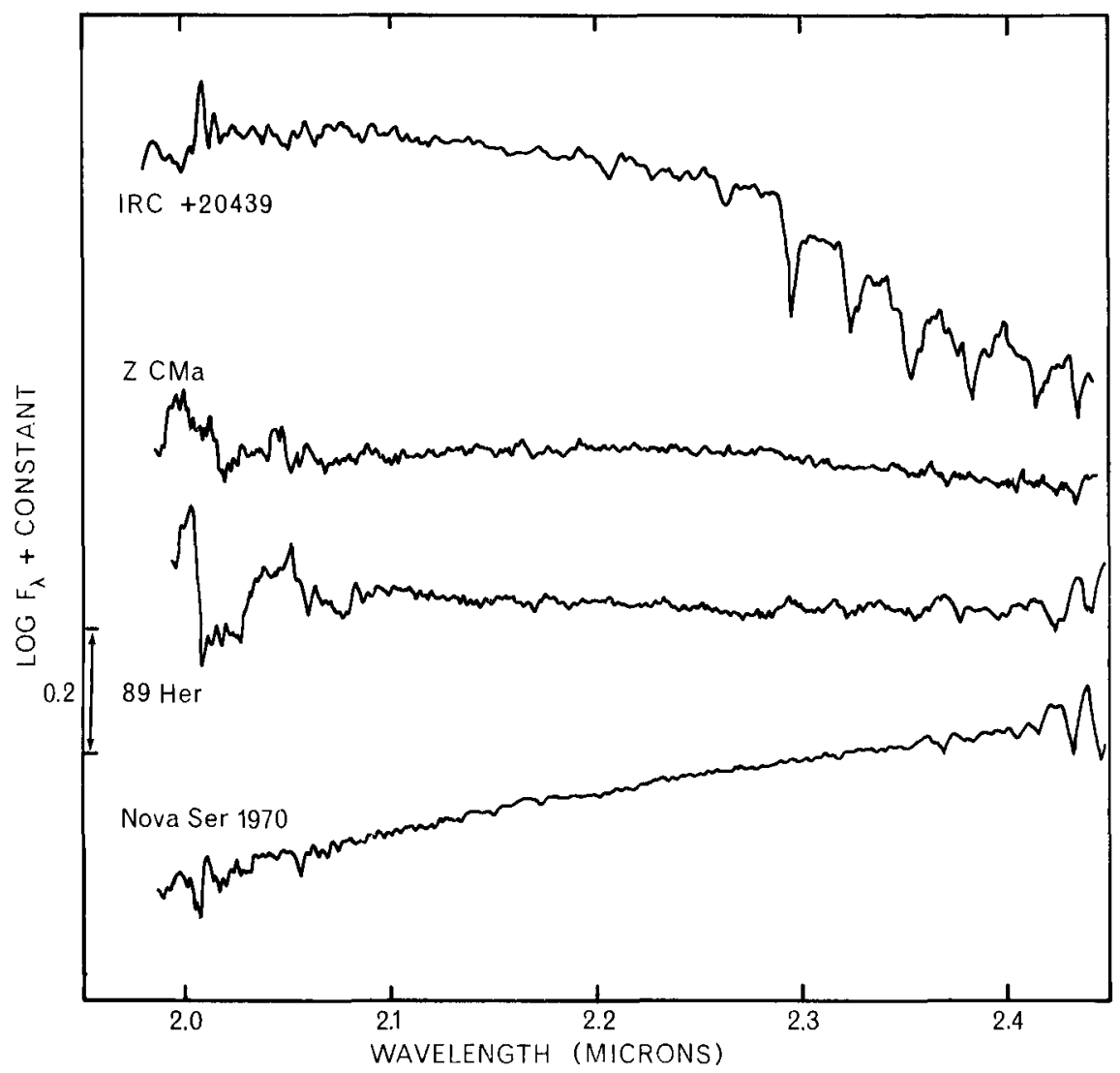

Fig. 8. Two micron spectra of four sources with early type optical components are shown. These are IRC +20439 , Z CMa, 89 Her and FH Ser (Nova Ser 1970), and are discussed in the text.

distribution close to $2.2 \mu$ indicates that there is a sizeable contribution from grains with temperatures $\sim 1300 \mathrm{~K}$. Neugebauer et al. (1971) have shown that a Larson (1969) type dust-cloud model where the dust opacity varies as $\lambda^{-1}$ gives a reasonable fit to the overall energy distribution.

\subsection{3. $89 \mathrm{Her}$}

This is an interesting F supergiant star with a large infrared excess (Gillett et al., 1970) which has also been interpreted in terms of emission from a circumstellar dust cloud. The high rate of mass loss is consistent with the formation of the dust cloud from the expelled material. The 2.0-2.5 $\mu$ spectrum presented by Gillett et al. (1970) is shown in Figure 8, and also shows a smooth dust like continuum. The most interesting feature of both the broad-band infrared and spectral observations of $89 \mathrm{Her}$, is their remarkable similarity to those of the carbon rich variable $\mathrm{R} \mathrm{CrB}$. Unlike the infrared excesses found in the $G$ supergiants (Humphreys et al., 1971) there is no evidence that the dust particles are composed silicates. Clearly further observa- 
tions are needed to determine the exact nature of the proposed grains around $89 \mathrm{Her}$.

\subsubsection{FH Ser (Nova Ser 1970)}

Our final example is the nova which underwent a remarkable infrared phase some 40-60 days following its optical outburst (Hyland and Neugebauer, 1970). This infrared phase was interpreted in terms of dust emission from grains $(T \sim 900 \mathrm{~K})$ found at large distances from the star. The infrared spectrum shown is that of Hyland and Neugebauer (1970) after correction for terrestrial absorption, and in common with $\mathrm{Z}$ CMa shows the remarkably smooth and featureless continuum characteristic of blackbody emission from grains. Unfortunately, this region provides no knowledge as to the nature of the grains such as is available from narrow band photometry in the $8-14 \mu$ region. Note that at the phase of observation no $\mathrm{B} \gamma$ nor $\mathrm{He} \lambda 2.058 \mu$ emission is seen in the spectrum of FH Ser.

The above four examples illustrate the diagnostic value of two micron spectroscopy for a variety of infrared sources.

\subsection{COMPARISON OF THE CONTINUOUS ENERGY DISTRIBUTION WITH MODEL ATMOSPHERES}

In the previous sections we have concentrated on the observations and interpretation of the molecular and atomic absorption features visible in the $2.0-2.5 \mu$ wavelength region. Implicit throughout the discussion has been the possibility of comparing the overall observed energy distribution with the predictions of model atmospheres. This procedure has been generally overlooked although it should be invaluable for testing the predictions of realistic model atmospheres of late type stars as they become available (Alexander and Johnson, 1972; Querci, F. \& M., 1974). It has been emphasized by Frogel (1971) that Auman's (1969) models including water vapour opacities do not agree with the observations, the predicted $\mathrm{H}_{2} \mathrm{O}$ bands being too strong. Future observational and theoretical studies of medium resolution spectra should include detailed comparisons of the observed and predicted fluxes in the manner which has proved highly successful in the interpretation of visual scans of early type stars.

\subsection{Stellar POPUlation StUdies in Galaxies and gLObular CluSters}

Knowledge of the spectral type - molecular band strength relations as discussed in Section 4 can be used in conjunction with observations to provide useful information on the cores of galaxies and globular clusters. Because these are generally faint in the two micron region it is necessary to reduce the resolution and/or to use a system of narrow band filters to define the molecular $\mathrm{CO}$ and $\mathrm{H}_{2} \mathrm{O}$ band strengths. A pioneering study along these lines has been performed by Baldwin et al. (1973). Using narrow band filters centered at 2.10,2.20 and $2.31 \mu$ they measured $\mathrm{CO}$ and $\mathrm{H}_{2} \mathrm{O}$ indices for the nuclear regions of the three galaxies M31, M81 and NGC 5195 in an effort to resolve the controversy regarding the luminosity function for cool stars. Comparison of the band strength with the standard relations for giants and dwarfs shows that all three galactic nuclei have band strengths similar to late type giant stars and precludes the existence of a dwarf enriched sequence $(M / L=44)$ such as has been proposed by 
Spinrad and Taylor (1971). This result is in agreement with Whitford's (1972) inability to detect the Wing-Ford $(9910 \AA)$ band in the nucleus of M31.

Studies of this nature appear to be very promising for future investigations of the stellar population content of galactic nuclei, elliptical galaxies and globular clusters and emphasize the versatility of spectroscopic observations in the two micron region.

\section{Conclusion}

Throughout this paper I have tried to show that medium resolution spectroscopy in the two micron region has a vital role to play in a variety of astrophysical problems. We have seen that the molecular CO band strengths can be used as temperature and luminosity indicators, while also being useful under certain conditions for the study of light element abundances and the ${ }^{12} \mathrm{C} /{ }^{13} \mathrm{C}$ isotope ratios. The strength of the $1.9 \mu$ band of $\mathrm{H}_{2} \mathrm{O}$ appears to be intimately related to the LPV M stars, and has lead to interesting deductions regarding $\mathrm{S}$ stars. Two micron spectroscopy was also shown to be valuable in defining the properties of infrared components of various stars with large infrared excesses.

The future of observations of this nature seems assured with its extension to use as an indicator of stellar content in the nuclei of galaxies and globular clusters, and the advent of new high sensitivity detectors will ensure that even more exciting domains may be explored.

\section{Acknowledgements}

It is a pleasure to acknowledge the assistance of many of the Cal Tech Infrared Group in obtaining the spectra discussed here. The work at Cal Tech was supported in part by NASA Grants Nos. NGL 05-002-007, NGL 50-002-207 and NSF Grant GP 9527.

\section{References}

Alexander, D. R. and Johnson, H. R.: 1972, Astrophys. J. 176, 629.

Auman, J. R.: 1969, Astrophys. J. 157, 799.

Baldwin, J. R., Danziger, I. J., Frogel, J. A. and Persson, S. E.: 1973, Astrophys. Letters 14, 1. Becklin, E. E., Frogel, J. A., Hyland, A. R., Kristian, J., and Neugebauer, G.: 1969, Astrophys. J. Letters 158, L133.

Bessell, M. S. and Lee, Y.: 1972, Proc. Astron. Soc. Australia 2, 154.

Boyce, P. B. and Sinton, W. M.: 1964, Astron. J. 69, 534.

Boyce, P. B. and Sinton, W. M.: 1965, Sky Telesc. 29, 78.

Cannon, R. D.: 1970, Monthly Notices Roy. Astron. Soc. 150, 111.

Eggen, O. J.: 1972, Astrophys. J. 174, 45.

Frogel, J. A.: 1970, Astrophys. J. Letters 162, L5.

Frogel, J. A.: 1971, Ph.D. Thesis, California Institute of Technology.

Frogel, J. A. and Hyland, A. R.: 1972, Mem. Soc. Roy. Sci. Liege, 6th Ser. 3, 111.

Gillett, F. C. and Stein, W. A.: 1971, Astrophys. J. 164, 77.

Gillett, F. C., Hyland, A. R., and Stein, W. A.: 1970, Astrophys. J. Letters 162, L21.

Hilgeman, T. W.: 1970, Ph.D. Thesis, California Institute of Technology.

Humphreys, R. M., Strecker, D. W., and Ney, E. P.: 1971, Astrophys. J. Letters 167, L35.

Hyland, A. R.: 1973 , in preparation.

Hyland, A. R. and Gingold, R. A.: 1972, Proc. Astron. Soc. Australia 2, 155. 
Hyland, A. R. and Neugebauer, G.: 1970, Astrophys. J. Letters 160, L177.

Hyland, A. R., Becklin, E. E., Neugebauer, G., and Wallerstein, G.: 1969. Astrophys. J. 158, 619. Hyland, A. R., Becklin, E. E., Frogel, J. A., and Neugebauer, G.: 1972, Astron. Astrophys. 16, 204. Johnson, H. L.: 1968, Astrophys. J. Letters 154, L125.

Johnson, H. L. and Méndez, M. E.: 1970, Astron. J. 75, 785.

Johnson, H. L., Coleman, I., Mitchell, R. I., and Steinmetz, D. L.: 1968, Comm. Lunar Planet. Lab. 7,83 .

Johnson, H. R., Marenin, I., and Price, S. D.: 1972, J. Quant. Spectrosc. Radiat. Transfer 12, 189. Kuiper, G. P.: 1962, Comm. Lunar Planet. Lab. $1,1$.

McCammon, D., Münch, G., and Neugebauer, G.: 1967, Astrophys. J. 147, 575.

Mendoza, E. E. and Johnson, H. L.: 1965, Astrophys. J. 141, 161.

Mertz, L.: 1965, Astron. J. 70, 548.

Moroz, V. I.: 1966, Soviet Astron. 10, 47.

Neugebauer, G. and Leighton, R. B.: 1968, Two-Micron Sky Survey - A Preliminary Catalog, NASA Sp-3047.

Neugebauer, G. and Westphal, J. A.: 1968, Astrophys. J. Letters 152, L89.

Neugebauer, G., Becklin, E. E., and Hyland, A. R.: 1971, Ann. Rev. Astron. Astrophys. 9, 67.

Querci, F. and Querci, M.: 1974, this volume, p. 341.

Racine, R., Becklin, E. E., Hyland, A. R., and Neugebauer, G.: 1971, data quoted by Neugebauer et al. (1971).

Schild, R. E. Peterson, D. M., and Oke, J. B.: 1971, Astrophys. J. 166, 95.

Sinton, W. M.: 1966, Astron. J. 71, 398.

Spinrad, H. and Wing, R. F.: 1969, Ann. Rev. Astron. Astrophys. 7, 249.

Spinrad, H. and Taylor, B. J.: 1971, Astrophys. J. Suppl. 22, 445.

Thompson, R. I.: 1974, this volume, p. 255.

Thompson, R. I. and Schnopper, H. W.: 1970, Astrophys. J. Letters 160, L97.

Thompson, R. I., Schnopper, H. W., and Rose, W. K.: 1971, Astrophys. J. 163, 533.

Thompson, R. I., Johnson, H. L., Forbes, F. F., and Steinmetz, D. L.: 1972, Publ. Astron. Soc. Pacific 84, 779 .

Whitford, A. E.: 1972, Bull. Am. Astron. Soc. 4, 230.

Wing, R. F.: 1974, this volume, p. 285.

Wing, R. F.: 1967, Ph.D. Thesis, University of California, Berkeley.

\section{DISCUSSION}

Thompson: Could it be that as the temperature goes down it is simply just the association of $\mathrm{C}_{2}$ and $\mathrm{CN}$, that reduces the $\mathrm{CO}$ first overtone bands not so much an increase in carbon as you thought, but possibly just that we are associating more molecules.

Hyland: You mean that would increase the total opacity?

Thompson: Right.

Hyland: That is possible, but I am not sure that it would give the magnitude of the effect. 